\title{
Screening for Autism Spectrum Disorder in Children up to Age 2.5 years in a Tertiary Care Hospital
}

Anum Farooq ${ }^{1}$, Shoaib Ahmed ${ }^{2}$

\begin{abstract}
Objective: To investigate the frequency of Autistic Spectrum Disorder (ASD) by screening 16 to 30 months old children, reporting to a tertiary care hospital.

Study Design: Cross sectional study.

Place and Duration of Study: The study was conducted in the Paediatrics Outpatient Department of Military Hospital, Rawalpindi from November 2017 to August 2018.

Materials and Methods: A total of 163 children between the age of 16-30 months were screened using Urdu translated version of Modified Checklist for Autism in Toddlers, revised with Follow-Up (M-CHAT-R/F ${ }^{\mathrm{TM}}$ ). Those who screened positive on MCHAT-R were assessed using Childhood Autism Rating Scale-2 (CARS 2) and Diagnostic and Statistical Manual-5 (DSM-5) as diagnostic tools.

Results: Among the 163 children screened with MCHAT, 33 were found positive for autism. The positive individuals were further assessed on CARS-2 and DSM-5; five children were diagnosed with ASD at moderate to severe level. This study has shown that $3 \%$ of children visiting the hospital for various reasons suffer from ASD. Conclusion: This study concluded that $3 \%$ of the children coming to the hospital for any purpose were found to have ASD. There is a need to make Level 1 screening an essential part of regular check-ups of children in Paediatrics outpatient clinics.
\end{abstract}

\section{Key Words: Autism Spectrum Disorder, Prevalence, Screening.}

How to cite this: Farooq A, Ahmed S. Screening for Autism Spectrum Disorder in Children up to Age 2.5 years in a Tertiary Care Hospital. Life and Science. 2020; 1(1): 11-15. doi: http://doi.org/10.37185/LnS.1.1.74

\section{Introduction}

Autism Spectrum Disorder (ASD) is a neurodevelopmental disorder that involves set of clinical phenotypes ${ }^{1}$ and a complex heterogeneous biological etiology. ${ }^{2}$ People with autism are characterised by persistent deficit in social interaction, communication and repetitive, restricted pattern of behaviours. It is proposed that autism occurs in about $1 \%$ of the general population. ${ }^{1}$ The prevalence of ASD in the United States of America (USA) is estimated to be $1.5 \%{ }^{3}$ and in South Asian countries, it ranges from 1.81 to $2.6 \% .{ }^{4,5}$ However, no authentic prevalence data on

${ }^{1}$ Department of Clinical Psychology

Shifa Tameer-e-Millat University, Islamabad

${ }^{2}$ Department of Paediatrics

Hearts International Hospital, Rawalpindi

Correspondence:

Ms. Anum Farooq

Department of Clinical Psychology

Shifa Tameer-e-Millat University, Islamabad

E-mail: anum.dcp@stmu.edu.pk

Funding Source: NIL; Conflict of Interest: NIL

Received: Sep 11, 2019; Revised: Dec 24, 2019

Accepted: Jan 07, 2020
ASD in Pakistan has been published so far. An estimate in a Pakistan Country Report survey (Government of NWFP, 2009) divulges that there is a minimum of 345,600 ASD cases. ${ }^{6}$ The absence of authentic prevalence studies is due to lack of resources, public awareness about the disorder and experts to conduct such epidemiological studies at the national level.

In the recent years, there has been a rise in the epidemiological studies which is due to enhanced awareness of autism as a spectrum of impairments and also elaboration in the diagnostic criteria of DSM 5. 7,89 Considering this diversity, various diagnostic and screening inventories were devised to identify the incidence and prevalence of the disorder in various populations. ${ }^{8,9}$ These standardized tools helped to estimate the current global trends of autism prevalence, which is 1 in 59 and this number is increasing drastically. ${ }^{10}$

Screening is a process used to monitor child development in the early age for further assessment, leading to the identification of affected cases and hence increased sensitivity and specificity of a 
screening instrument. ${ }^{11}$ It is believed that the early identification of autism through screening results in early management and better prognosis. ASD can be diagnosed as early as 18 months to 2 years by an experienced professional. ${ }^{12}$ However, in most of the cases the child is not diagnosed till he gets older. In Pakistan, the identification, screening and diagnosis of ASD by health care professionals is itself a matter of grave concern. ${ }^{13}$ Hospitals (or) clinical setups are the first point of contact for infants for vaccination and other regular check-ups but they lack standard protocol or guidelines to diagnosis and intervene for children with ASD. ${ }^{14}$

Screening done by standardized tools, makes the process more effective. The American Academy of Paediatrics (AAP) guidelines recommend Modified Checklist for Autism in Toddlers as an authentic and widely used screening tool. ${ }^{15}$ As per AAP guidelines, routine screening of toddlers and infants using standardized instruments should be incorporated in Paediatrics departments of every hospital. The screening for developmental delays should be done in first 2 years at frequent intervals $(9,18,24$ or 30 months). ${ }^{15}$ Research by Chlebowski et al provides empirical support for the use of the M-CHAT in primary care settings. ${ }^{16}$

Considering the rise of ASD worldwide, the United States Preventive Services Task Force recommends ASD screening for all children below the age of 3 years. ${ }^{17}$ It is evident that Level 1 screening in primary care setting is pertinent to identify ASD in children, and to develop sound protocols for referrals and management. ${ }^{17}$

However, health care facilities in Pakistan lack such screening services as a result exact prevalence of ASD in our population cannot be estimated. The study was conducted to estimate the frequency of the ASD among children 16-30 months attending paediatric OPD.

\section{Materials and Methods}

The survey was conducted at a tertiary care hospital, Outpatient Paediatrics Department at Military Hospital Rawalpindi for a period of ten months from November 2017 to August 2018. Every child coming to the children's OPD for a medical visit was screened for ASD.

The study population consisted of all children aged 16-30 months, who visited the Children OPD for various health purposes.

Most of the screened cases were males (67.5\%) with majority of them in the age category of 26-30 months $(43 \%)$, living in a nuclear family (54.6\%).

Many of the mothers were aged between 26 to 30 years (39\%) at the time of child's birth. Most of them reported no complications during pregnancy (69\%). Among those who reported complication (29\%), anaemia was a major complication. Most of the children were born at 9 months $(86.5 \%)$ with birth weight of 5-7 pounds (61.3\%) without any complications during birth (89.6\%) (Table 1).

\begin{tabular}{|c|c|c|}
\hline Gender & $\mathbf{n}$ & $\%$ \\
\hline Male & 110 & $67.5 \%$ \\
\hline Female & 53 & $32.5 \%$ \\
\hline \multicolumn{3}{|l|}{ Age of the Child } \\
\hline $16-20$ & 45 & $27 \%$ \\
\hline $21-25$ & 47 & $28 \%$ \\
\hline $26-30$ & 71 & $43 \%$ \\
\hline \multicolumn{3}{|l|}{ Family System } \\
\hline Nuclear & 89 & $54.6 \%$ \\
\hline Joint & 74 & $45.3 \%$ \\
\hline \multicolumn{3}{|c|}{ Mother's Age at Time of Birth } \\
\hline $20-25$ years & 48 & $29 \%$ \\
\hline $26-30$ years & 64 & $39 \%$ \\
\hline $31-35$ years & 18 & $11 \%$ \\
\hline $36-40$ years & 3 & $1 \%$ \\
\hline Missing cases & 30 & $18.4 \%$ \\
\hline \multicolumn{3}{|c|}{ Physical Illness during Pregnancy } \\
\hline Yes & 48 & $29 \%$ \\
\hline No & 115 & $70.5 \%$ \\
\hline \multicolumn{3}{|c|}{ Medical Issue during Pregnancy } \\
\hline Anaemia & 22 & $13.4 \%$ \\
\hline Bleeding in first trimester & 6 & $3.68 \%$ \\
\hline Gestational diabetes & 4 & $2.45 \%$ \\
\hline Missing cases & 131 & $80.3 \%$ \\
\hline \multicolumn{3}{|l|}{ Pregnancy Duration } \\
\hline 9 months & 142 & $87.1 \%$ \\
\hline Before 9 months & 19 & $11.7 \%$ \\
\hline After 9 months & 2 & $1.2 \%$ \\
\hline \multicolumn{3}{|l|}{ Child's Birth Weight } \\
\hline $5.0-7.0$ pounds & 100 & $61.3 \%$ \\
\hline $7.1-9.0$ pounds & 39 & $23.9 \%$ \\
\hline Missing cases & 24 & $14.7 \%$ \\
\hline \multicolumn{3}{|l|}{ Complications during Birth } \\
\hline Yes & 17 & $10.4 \%$ \\
\hline No & 146 & $89.6 \%$ \\
\hline
\end{tabular}

Data Collection Procedure

Quantitative screening and diagnostic questionnaire survey were carried out of all the eligible cases in OPD. Initial screening was done with M-CHAT RF and those who scored above the cutoff score (cutoff score $>2$ ) were assessed later on CARS-2 and DSM-5 ASD criteria. 
Keeping in view the lack of insight/cognitive capacity of the children to give consent for the research, permission was taken from the parent or legal guardian.

\section{Assessment Tools}

1. Modified Checklist for Autism in Toddlers, Revised with Follow-Up

The Modified Checklist for Autism in Toddlers, revised with Follow-Up (M-CHAT-R/F) ) is a twostage parent report screening tool, that is used to assess the risk of ASD. ${ }^{18}$ The primary goal of $\mathrm{M}$ CHAT-R/F is to maximize sensitivity as a Level 1 screening tool or to assess the risk for ASD. The tool is valid to screen toddlers between age 1630 months. MCHAT also screens children with other neurodevelopmental disorders. An Urdu translated version of this screening tool was used which had been previously translated and validated. ${ }^{19}$

Scoring of MCHAT-RF: ${ }^{18}$

Low-Risk: If the score is between 0-2 and the child is younger than 24 months then further screening is needed after second birthday.

Medium-Risk: A total score in the range of 3-7 requires a follow up screening first. If the score remains more than 2 , the child is considered to be positive and diagnostic evaluation should be done.

High-Risk: A score in the range of 8-20 requires diagnostic evaluation immediately.

2. Childhood Autism Rating Scale- Second Edition (CARS-II)

Childhood Autism Rating Scale for assessment and diagnosis of children with ASD was used for post assessment. ${ }^{20}$ It is one of the most widely used, reliable and valid tool for assessing behaviors associated with autism. ${ }^{21,22}$ The child is quantifiably rated by the clinician (based on direct observation) on 15 behaviors to determine symptom severity. The scale is used for children aged 2 years and above. The tool has high reliability with DSM 5 diagnostic criteria of autism. ${ }^{23}$

Severity groups based on CARS 2 are given in Table 2. ${ }^{21}$

3. Diagnostic and Statistical Manual-5 (DSM-5)

ASD is diagnosed on persistent deficit in social communication, interaction and repetitive or

\begin{tabular}{lll}
\hline \multicolumn{2}{l}{ Table 2: Severity Groups } \\
\hline ASD Severity Level & $\begin{array}{l}\text { Raw Score } \\
\text { Range }\end{array}$ & $\begin{array}{l}\text { Raw Score Range } \\
\text { for Age 13+ }\end{array}$ \\
$\begin{array}{l}\text { Minimal to no } \\
\text { symptoms }\end{array}$ & $15-29.5$ & $15-27.5$ \\
$\begin{array}{l}\text { Mild to moderate } \\
\text { symptoms } \\
\text { Severe symptoms }\end{array}$ & $30-36.5$ & $28-34.5$ \\
\hline
\end{tabular}

restrictive behaviors. The severity is based on the dysfunction in communication, interaction and fixation on repetitive or restricted behaviors thus defining the amount of support required by the child. ${ }^{1}$

Severity level 1: "Requiring support"

Severity Level 2: "Requiring substantial support"

Severity Level 3: "Requiring very substantial support"

\section{Results}

In the present study, 163 cases aged 16-30 months were screened using Urdu translated $\mathrm{MCHAT} \mathrm{RF}{ }^{\mathrm{TM}}$ in the Paediatric OPD of Military Hospital (tertiary healthcare). A total of 33 children screened positively on the M-CHAT checklist. Screened cases (cutoff $>2$ ) were assessed on CARS-2 and DSM-5. Five cases were identified with moderate to severe level of ASD. These children were diagnosed according to DSM-5 ASD diagnostic criteria. The prevalence rate of ASD among children aged 16-30 months in our study was $3 \%$. Out of five cases, three were diagnosed with moderate and two with severe ASD. Out of 33 cases, 18 screened on MCHAT had different developmental disabilities.

\section{Discussion}

The worldwide prevalence of autism is 1 in $59^{10}$ and it is reported to be $1 \%$ in the general population. ${ }^{1}$ The prevalence estimated around the world shows the endemic nature of this disorder. In recent years, developed countries are monitoring autism prevalence through systematic early screening processes, but in developing countries in general, so far very few studies have been done on prevalence at a large scale. ${ }^{4}$ This might be due to lack of economic resources, experts, and public awareness about the developmental disorders.

Our study results are similar to the few studies done in Pakistan on paediatric population. A previous study in a tertiary health care unit of Rawalpindi reported that $8.6 \%$ of the patients screened in adult psychiatric ward had diagnosis of ASD which is a 
considerably high percentage. ${ }^{24}$ According to the global trend in 2010, the estimated ASD population was 52 million with 1 in 132 persons diagnosed with ASD. ${ }^{25}$ This number has drastically increased to 1 in 59 (2018 reporting) ${ }^{10}$, while New Jersey's autism rate is reported to be $3 \% .{ }^{10}$ Our study showed relatively similar frequency compared to the global trends observed in the general population. ${ }^{26}$ Moreover, it is also consistent with the trends shown in a few national studies done in tertiary care setups. The local literature related to prevalence is limited to hospital-based populations or children with autism in special schools ${ }^{27-32}$ and therefore it is difficult to generalize these results to other settings. As there no authenticated autism prevalence data available for Pakistan, the study highlights the need to acquire accurate and reliable estimates of autism prevalence at the national level.

The current study demonstrates the feasibility of Level 1 screening for ASD in the tertiary care setting, highlighting the significance and relevance of using standardized screening tools like MCHAT in early detection of ASD and establishing Level 1 screening in tertiary care settings. ${ }^{11}$ This can be done by making early screening part of regular child checkups at an early age in hospitals as per American Academy of Pediatrics (AAP) guidelines. This will help in early identification of ASD, better management and prognosis and possibly less financial and emotional burden on the families.

The study has limitation that it used CARS-2 as a diagnostic tool, further studies are needed using Autism Diagnostic Observation Schedule (ADOS) to diagnose children with ASD.

\section{Conclusion}

This study has shown that $3 \%$ of children visiting hospitals for various reasons suffer from ASD. It highlights the need to make Level 1 screening as an essential part of regular checkup of children in outpatient Pediatrics wards.

\section{Acknowledgements}

Prof. Dr. Syed Muhammad Imran Majeed, Dr. Arif Mahmud, Ms. Fizza Haider and Ms. Taskeen Mansoor participated in technical editing of the manuscript and proof reading.

Prof. Dr. Mujtaba Quadri critically reviewed the study proposal.

\section{REFERENCES}

1. Diagnostic and Statistical Manual of Mental Disorders: DSM-5 (5th edition) Washington, DC American Psychiatric Association; 2013.

2. Coleman M, Gillberg C. The Autisms. New York: Oxford University Press. 2012; 39: 853-64.

3. Centers for Disease Control and Prevention. 2010 (cited $15^{\text {th }}$ August 2019). Diseases and conditions. Available from https://www.cdc.gov/ncbddd/Autism/index.html.

4. Kawamura Y, Takahashi O, Ishii T. Re-evaluating the incidence of pervasive developmental disorders: Impact of elevated rates of detection through implementation of an integrated system of screening in Toyota, Japan. Psychiatry and Clinical Neurosciences. 2008; 62: 152-9.

5. Kim Y, Leventhal B, Koh Y, Fombonne E, Laska E, Lim E, et al. Prevalence of Autism Spectrum Disorders in a Total Population Sample. American Journal of Psychiatry. 2011; 168: 904-12.

6. Pakistan Country Report-Autism. Social Welfare and Women Development Department, Government of NWFP; 2009. Available from http://www.nise.go.jp/kenshuka /josa/kankobutsu/pub_d/d-292/d-292_19.pdf.

7. Fombonne E. Epidemiology of Pervasive Developmental Disorders. Pediatric Research. 2009; 65: 591-8.

8. King $M$, Bearman P. Diagnostic change and the increased prevalence of autism. International Journal of Epidemiology. 2009; 38: 1224-34.

9. Rice C, Rosanoff M, Dawson G, Durkin M, Croen L, Singer A, et al. Evaluating changes in the prevalence of the Autism Spectrum Disorders (ASDs). Public Health Reviews. 2012; 34: 1-22.

10. Centers for Disease Control and Prevention (CDC). 2018 (cited $15^{\text {th }}$ August 2019). Disease and condition. Available from https://www.autismspeaks.org/science-news/cdcincreases-estimate-autisms-prevalence-15-percent-1-59children.

11. Robins D. Screening for autism spectrum disorders in primary care settings. Autism. 2008; 12: 537-56.

12. Autism Speaks. 2018 (cited 10 August 2019). Available from: https://www.Autismspeaks.org/provider/earlyidentification-diagnosis-asd.

13. Imran N, Chaudry M, Azeem M, Bhatti M, Choudhary Z, Cheema M. A survey of Autism knowledge and attitudes among the healthcare professionals in Lahore, Pakistan. BMC Paediatrics. 2011; 11: 107.

14. Shah K. What Do Medical Students Know about Autism? Autism. 2001; 5: 127-33.

15. Zwaigenbaum L, Bauman M, Fein D, Pierce K, Buie T, Davis P, et al. Early Screening of Autism Spectrum Disorder: Recommendations for Practice and Research. Pediatrics. 2015; 136: S41-S59.

16. Chlebowski C, Robins D, Barton M, Fein D. Large-Scale Use of the Modified Checklist for Autism in Low-Risk Toddlers. Pediatrics. 2013; 131: e1121-e7.

17. The Centers for Disease Control and Prevention (CDC). 2016 (cited 10 August 2019). Screening and Diagnosis of Autism Spectrum Disorder for Healthcare Providers. Available from https://www.cdc.gov/ncbddd/autism/hcp-screening.html. 
18. Robins DL, Fein D, Barton M. The Modified Checklist for Autism in Toddlers, Revised with Follow-Up (M-CHAT-R/F). 2009.

19. Sadia H, Farooq A. Urdu translation of The Modified Checklist for Autism in Toddlers, Revised with Follow-Up ( M-CHAT-R/F). 2018. Available from https://mchatscreen.com/mchat-rf/translations/.

20. Schopler E, Van Bourgondien ME, Wellman GJ, Love SR. The Childhood Autism Rating Scale (2nd ed.) (CARS2). 2010. Los Angeles, CA: Western Psychological Services.

21. Chlebowski C, Green J, Barton M, Fein D. Using the Childhood Autism Rating Scale to Diagnose Autism Spectrum Disorders. Journal of Autism and Developmental Disorders. 2010; 40: 787-99.

22. Russell P, Daniel A, Russell S, Mammen P, Abel J, Raj L, et al. Diagnostic accuracy, reliability and validity of Childhood Autism Rating Scale in India. World Journal of Pediatrics. 2010; 6: 141-7.

23. Park E, Kim J. Factor structure of the Childhood Autism Rating Scale as per DSM-5. Pediatrics International. 2015; 58: 139-45.

24. Khan S, Qayyum R, Iqbal J. Prevalence of Autism Spectrum Disorders (ASD) and Attention Deficit Hyperactivity Disorders (ADHD) among adult psych. Pakistan Armed Forces Medical Journal. 2019; 69: 419-23.

25. Baxter AJ, Brugha T, Erskine H, Scheurer RW, Vos T, Scott JG. The epidemiology and global burden of Autism spectrum disorders. Psychol Med. 2014; 45: 601-13.

26. Elsabbagh M, Divan G, Koh Y, Kim Y, Kauchali S, Marcín C, et al. Global Prevalence of Autism and Other Pervasive Developmental Disorders. Autism Research. 2012; 5: 16079.

27. Syed EU, Hussein SA, Yousafzai AW. Developing services with limited resources: establishing a CAMHS in Pakistan. Child Adolesc Mental Health. 2007; 12: 121-4.

28. Tareen A, Mirza I, Minhas A, Minhas F, Rahman A. Developing a child and adolescent mental health service in a low-income country: A global partnership model. Psychiatr Bull. 2009; 33: 181-3.

29. Sarwat A, Ali SI, Ejaz MS. Mental health morbidity in children: a hospital based study in child psychiatry clinic. Pak J Med Sci. 2009; 25: 982-5.

30. Imran N, Bhatti MR, Anwar A, Najmi F, Haider II. Children's mental health: Pattern of referral, distribution of disorders and service use in child psychiatry outpatient setting. Pak J Med Sci. 2012; 28: 22-6.

31. Imran N, Azeem MW, Sattar A, Bhatti MR. Frequency of ICD10 psychiatric diagnosis in children with intellectual disability in Lahore, Pakistan \& caregiver's perspective. Pak J Med Sci. 2015; 31: 285-9.

32. Suhail K, Zafar F. Prevalence of autism in special education schools of Lahore. Pakistan Journal of Psychological Research. 2008; 23: 45-64. 